Article

\title{
Multi-Functional Magnetic Photoluminescent Photocatalytic Polystyrene-Based Micro- and Nano-Fibers Obtained by Electrospinning
}

\section{Michel Schaer ${ }^{1}$, Mireille Crittin ${ }^{2}$, Lamia Kasmi ${ }^{2}$, Katarzyna Pierzchala ${ }^{2}$, Caroline Calderone ${ }^{3}$,} Reinaldo G. Digigow ${ }^{4,5}$, Alke Fink ${ }^{4,5}$, László Forró ${ }^{2}$ and Andrzej Sienkiewicz ${ }^{2, *}$

1 Institute of Materials, School of Engineering, EPFL, Lausanne CH-1015, Switzerland; E-Mail: michel.schaer@epfl.ch

2 Institute of Physics of Condensed Matter, School of Basic Sciences, EPFL, Lausanne CH-1015, Switzerland; E-Mails: mireille.crittin@gmail.com (M.C.); kasmil@phys.ethz.ch (L.K.); katarzyna.pierzchala@epfl.ch (K.P.); laszlo.forro@epfl.ch (L.F.)

3 Interdisciplinary Center for Electron Microscopy, School of Basic Sciences, EPFL, Lausanne CH-1015, Switzerland; E-Mail: caroline.calderone@epfl.ch

4 Adolphe Merkle Institute, University of Fribourg, Fribourg CH-1723, Switzerland; E-Mails: reinaldog.digigow@unifr.ch (R.G.D.); alke.fink@unifr.ch (A.F.)

5 Department of Chemistry, University of Fribourg, Fribourg CH-1700, Switzerland

* Author to whom correspondence should be addressed; E-Mail: andrzej.sienkiewicz@epfl.ch; Tel.: +41-21-693-43-37; Fax: +41-21-693-44-70.

Received: 30 November 2013; in revised form: 27 January 2014 / Accepted: 6 February 2014 / Published: 25 February 2014

\begin{abstract}
This work reports on the implementation of electrospinning (ES) as a facile route to encapsulate nano-engineered materials in a polystyrene (PS) matrix. We applied ES to co-encapsulate two kinds of nanoparticles, i.e., upconversion nanophosphors (UCNPs) and superparamagnetic iron oxide nanoparticles (SPIONs), in polystyrene (PS)-based micro- and nano-fibers (PSFs). This approach made it possible to integrate near-infrared (NIR) light-sensitive 500-nm $\quad \beta-\mathrm{NaYF}_{4}: \mathrm{Yb}, \quad$ Er UCNPs with 10-nm $\gamma-\mathrm{Fe}_{2} \mathrm{O}_{3}$ SPIONs in PS fibers. During the ES process, PSFs were additionally loaded with a well-established singlet oxygen $\left({ }^{1} \Delta_{\mathrm{g}}\right)$ photosensitizer, rose bengal (RB). The thus obtained PSFs revealed the promising features of prospective multi-functional magnetic photoluminescent photocatalytic nano-constructs.
\end{abstract}


Keywords: nanofibers; nano-encapsulation; electrospinning; superparamagnetic iron oxide nanoparticles; upconversion nanoparticles; singlet oxygen; near-infrared light

\section{Introduction}

There is a growing interest in designing composite nanostructured materials made of inorganic nanoparticles entrapped in organic polymer matrices [1]. In particular, encapsulation of inorganic nanoengineered particles (NPs) in a polymer matrix is of a high importance in various fields of applications, such as cosmetics, paints, additives, bio-medicine, etc. [2]. In this regard, numerous polymer-based encapsulation techniques have been reported using different approaches to encapsulate various inorganic NPs, like silica, titania, alumina, calcium carbonate, carbon black, magnetic iron oxides, metal nanoparticles and quantum dots [3-6]. The controlled fabrication of well-defined micro- and nano-sized composites encapsulated in polymer matrices has become one of the main topics in materials science oriented towards bio-medical applications. In particular, polymer-based fibrillar nanostructured composites provide a connection between the nanoscale world and the macroscale world, because their diameters and encapsulated active components are in the nanometer range, whereas their overall lengths are in the kilometer range [7]. Fabrics designed around polymer-based nano-fibrillar composites offer a plethora of interesting properties, such as exceptionally high surface area, ease of functionalization via simple chemistry and multi-functionality, just to name a few of them. These features are of particular importance for the design and fabrication of multi-functional biomaterials, which are necessary for advanced study in bio-sensing, tissue engineering and regenerative medicine [8]. Welldefined multi-functional nanostructured fibers are also finding applications in controlled drug delivery and drug release, nano-micro-electromechanical systems, advanced filtration, etc. [9].

Electrospinning (ES) is gaining increasing attention as a versatile method of preparing polymer-based micro- and nano-fibers, as well as a facile route towards the encapsulation of nanoscale materials [10]. ES is a polymer processing technique that uses electrostatic forces to uniaxially stretch a viscoelastic jet derived from a polymer solution (or polymer melt) and delivered through a millimeterscale nozzle to produce continuous nanometric and micrometric fibers, which are typically assembled into non-woven mats [11,12].

With respect to other nanofiber fabrication technologies, like template synthesis, self-assembly or phase separation, ES offers several advantages, including the simplicity and low-cost of the manufacturing routes, the possibility of using a great variety of polymers, both synthetic and natural, which can be processed into fibers, as well as a fairly good control of fiber mechanical properties and dimensions. ES makes it also possible to precisely monitor the fiber porosity and fiber orientation in the resulting mat. Most importantly, from the standpoint of bio-medical applications, functionalization of electrospun (E-spun) fibers can easily be integrated during their production [13,14].

Over the last two decades, ES has proven to be a versatile technological route towards obtaining multifunctional polymeric nanofibers suitable for numerous biomedical applications, including drug delivery and tissue engineering [15-17]. Reports are available on E-spun multifunctional polymer nanofibers containing visible light responsive dyes, like porphyrin or phthalocyanine, which can produce 
electronically excited molecular oxygen (singlet oxygen, ${ }^{1} \Delta_{\mathrm{g}}$ ) upon illumination with visible light and reveal an antibacterial effect [18-20]. Such multifunctional nanofibrillar composites have been found to be suitable for the fabrication of filters for water disinfection and the preparation of wound dressing patches to combat microbial infections.

In recent years, applications of upconversion nanophosphors (UCNPs) in bio-imaging and bio-sensing have been rapidly growing. Upconversion refers to a nonlinear optical processes characterized by the successive absorption of two or more low-energy photons via intermediate long-lived energy states, which is then followed by emission at a shorter wavelength than that of excitation. Thus, UCNPs, generally in the form of nano-/micro-crystals of lanthanide-doped rare earth fluorides, which utilize near-infrared (NIR) light rather than ultraviolet and visible light excitation, offer numerous advantageous features in bio-imaging, including increased penetration depth of biological tissues, large anti-Stokes shifts of the emitted light, sharp emission bandwidths, minimized autofluorescence and low photodamage [21,22]. Compared to traditional organic fluorophores or semiconductor quantum dots (QDs) that are currently used in bio-imaging, UCNPs exhibit much higher photostability and the absence of blinking and photobleaching [23,24]. Moreover, unlike QDs-based multi-photon bio-labels, which require simultaneous absorption of several NIR photons from a high-power pulsed laser source, UCNPs generate efficient upconversion luminescence (UCL) by a continuous-wave NIR diode laser, due to their real ladder-like energy levels of trivalent lanthanide ions [25]. Until quite recently, the low UCL efficiency of small-sized nanophosphors $(<50 \mathrm{~nm})$, as compared to their bulk crystal counterparts, has been a key problem in bio-analytical applications of UCNPs [22,26]. However, the most recent rapid advances in preparation methods of lanthanide-doped rare earth fluorides have led to the development of small-sized UCNPs, which exhibit both high UCL efficiency and photostability. Such UCNPs are now becoming available for biological labeling and imagining [27-30].

Among the broad spectrum of nano-engineered materials, magnetic nanoparticles (e.g., iron, iron oxides, cobalt and nickel oxides) are gaining increasing attention for many technological and biomedical applications, including magnetic storage media, bio-sensing, targeted drug delivery, contrast agents in magnetic resonance imaging (MRI) or hyperthermia [31,32]. In these applications, superparamagnetic iron oxide nanoparticles (SPIONs), like magnetite $\left(\mathrm{Fe}_{3} \mathrm{O}_{4}\right)$ or maghemite $\left(\gamma-\mathrm{Fe}_{2} \mathrm{O}_{3}\right)$, are widely encountered, because of their low toxicity and biocompatibility. In the context of designing magnetic nanocomposites, it has recently been demonstrated that the ES technology provides a flexible and costeffective way towards obtaining polymer-based multi-functional magnetic nanostructured fibers [33,34]. It has also been shown that E-spun nanocomposite magnetic fibers, which incorporate superparamagnetic SPIONs into fibrous polymeric scaffolds, open new avenues for novel biomedical applications, such as, e.g., magnetic field-stimulated bone tissue regeneration or hyperthermia treatment $[35,36]$.

In this work, we implemented the ES technology as a facile route to encapsulate two kinds of nano-engineered materials, i.e., UCNPs and SPIONs, in a polystyrene (PS) matrix. To this end, we implemented as UCNPs the commercially available particles of the hexagonal crystal phase of sodium yttrium fluoride $\left(\mathrm{NaYF}_{4}\right)$ doped with $\mathrm{Yb}^{3+}$ and $\mathrm{Er}^{3+}\left(\beta-\mathrm{NaYF}_{4}\right.$ : $\mathrm{Yb}$,Er). It is commonly accepted that the $\beta-\mathrm{NaYF}_{4}$ matrix exhibits the highest UCL efficiency among the lanthanide-doped fluorides [37]. In particular, under excitation with NIR light, $\beta-\mathrm{NaYF}_{4}$ :Yb,Er UCNPs reveal strong UCL emission bands in the green and red portions of the visible spectrum [38]. The UCNPs were co-encapsulated with the 
custom-synthesized $\gamma$ - $\mathrm{Fe}_{2} \mathrm{O}_{3}$ SPIONs in the polystyrene-based matrix in the same one-step ES process. This approach made it possible to obtain NIR light and magnetic field responsive polystyrene-based micro- and nano-fibers (PS-based fibers).

Moreover, in the same single-step ES process, PS-based fibers were additionally loaded with a singlet oxygen $\left({ }^{1} \Delta_{\mathrm{g}}\right)$ photosensitizer, rose bengal (RB). RB, a xanthene derivative among the most efficient producers of singlet oxygen, absorbs visible light in the range from 450 to $600 \mathrm{~nm}$, with a maximum around $550 \mathrm{~nm}$ [39]. Under excitation with visible light, RB forms long-lived excited triplet states, which can effectively be quenched by molecular oxygen to produce ${ }^{1} \Delta_{\mathrm{g}}$ with a high quantum yield, $\Phi_{\Delta}$, of 0.75 in water [40]. The perfect overlap of the green UCL emission and RB absorption in the green spectral range clearly points to the rationale of using $\mathrm{RB}$ as a ${ }^{1} \Delta_{\mathrm{g}}$ photosensitizer in conjunction with $\beta$ $\mathrm{NaYF}_{4}: \mathrm{Yb}, \mathrm{Er}$ UCNPs [41].

The nano-structured morphology of the thus produced PS-based fibers was characterized by scanning electron microscopy (SEM) and atomic force microscopy (AFM). The upconversion luminescence (UCL) properties of PS-based fibers under NIR light excitation were verified using spectrofluorimetry. The magnetic properties of PS-based fibers were checked with the electron spin resonance (ESR) technique. Finally, the photodynamic efficiency of PS-based fibers towards the generation of ${ }^{1} \Delta_{\mathrm{g}}$ in aqueous media under NIR light excitation was confirmed by reactive scavenging with 2,2,6,6-tetramethyl-4-piperidinol (TMP-OH), a widely-used ${ }^{1} \Delta_{\mathrm{g}}$ scavenger, which was followed by ESR detection of the resulting paramagnetic product, 4-hydroxy-2,2,6,6-tetramethylpiperidine-1-oxyl (TEMPOL). Overall, the obtained polystyrene-based fibers (PSFs) revealed promising features, such as prospective multi-functional magnetic photoluminescent photocatalytic nano-constructs.

\section{Experimental Section}

\subsection{UCNPS}

The commercial upconversion particles, product code PTIR 550/F (PTIR 550/F), were purchased from Phosphor Technology, Ltd., Stevenage, England. These materials consist of a powdered phosphor, $\beta$ $\mathrm{NaYF}_{4}: \mathrm{Er}, \mathrm{Yb}$, with average grain sizes in the range of 2.6-6.1 $\mu \mathrm{m}$ [42] and a high absolute upconversion quantum yield of $c a$. 3\%, as compared to particles of smaller sizes [26]. Under excitation with NIR light $\left(\lambda_{\mathrm{ex}}=975 \mathrm{~nm}\right)$, PTIR 550/F emits strong and narrow UCL emission bands at 520-550 nm and 650-670 $\mathrm{nm}$.

The as-received PTIR 550/F material was too large in average diameter to be employed in the process of ES. Therefore, prior to any further processing, PTIR550/F was ground in a ball mill. The dynamic light scattering (DLS) measurements performed on the ground material pointed to the achieved average particle sizes in the range of 300-550 nm. The TEM images of the as-received and ground PTIR 550/F, as well as the corresponding particle size distributions are shown in Figure 1. 


\subsection{SPIONs}

The superparamagnetic iron oxide nanoparticles, i.e., 10-nm $\gamma-\mathrm{Fe}_{2} \mathrm{O}_{3}$ SPIONs, were custom-synthesized using a modified method reported by Bee et al. [43] and van Ewijk et al. [44]. SPIONs were prepared by alkaline co-precipitation of ferric and ferrous chlorides in aqueous solution, as described in detail by Chastellain et al. [45]; a representative transmission electron microscopy (TEM) image is shown in Figure 2. This synthesis route yielded a concentrated suspension of SPIONs (10 mg Fe/mL) stabilized at acidic $\mathrm{pH}\left(\mathrm{HNO}_{3}, 10^{-2} \mathrm{M}\right)$.

Figure 1. The TEM images obtained for the commercial upconversion particles, PTIR 550/F, before (a) and after grinding (b) and the corresponding DLS-measured particle size distributions (c).
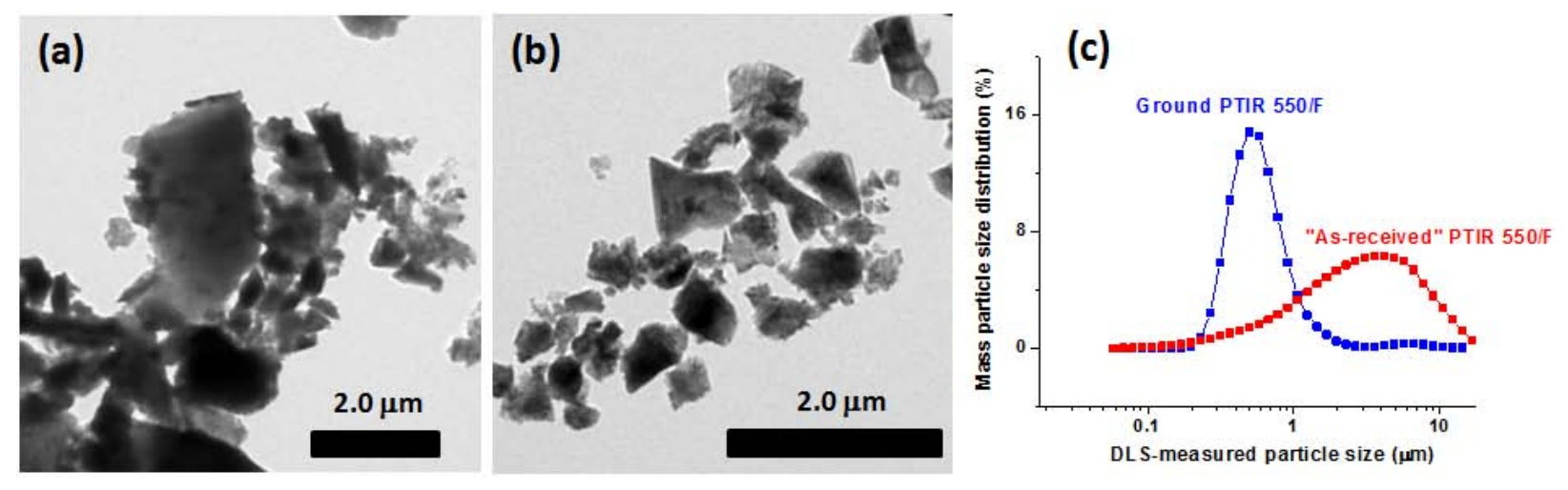

Figure 2. The TEM image of the custom-synthesized 10-nm $\gamma-\mathrm{Fe}_{2} \mathrm{O}_{3}$ superparamagnetic iron oxide nanoparticles (SPIONs).

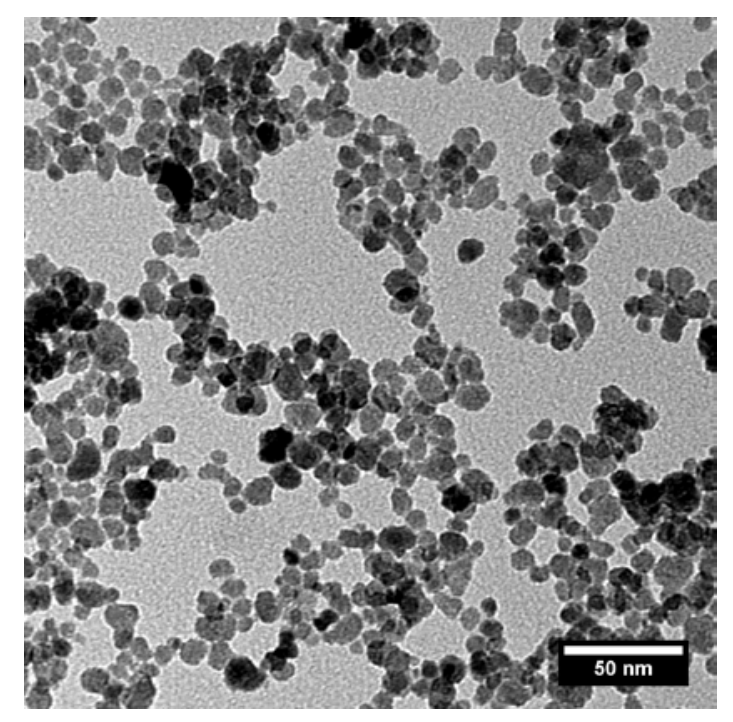

\subsection{Preparation of PS Solutions}

Pellets of white-colored PS (molecular weight $=192,000 \mathrm{~kg} / \mathrm{kmol}$, Sigma Aldrich) were dissolved in a 1:1 mixture of acetone and cyclohexane to a concentration of $20 \mathrm{wt} \%$. Subsequently, the nanomaterials, i.e., UCNPs and SPIONs, were added to the PS solutions. The following proportions were used: UCNPs, $0.7 \mathrm{mg} / \mathrm{mL}$ of dissolved PS (5 wt\%); SPIONs, $0.1 \mathrm{~mL}$ of the custom-made ferro-fluid per 
$1 \mathrm{~mL}$ of dissolved PS. Finally, RB (dye content of 95\%, Sigma Aldrich, Switzerland) dissolved in ethanol/acetone was added to the PS solution to get a concentration of $c a .0 .1 \mathrm{mg} \mathrm{RB} / \mathrm{mL}$ of the final PS solution (i.e., $\sim 100 \mu \mathrm{M}$ ). To ensure a good homogeneity of the prepared melts, the PS solutions were shaken and magnetically stirred for a prolonged time (usually more than $12 \mathrm{~h}$ ).

\subsection{ES Apparatus}

The polystyrene-based fibers (PSFs) were produced by the ES process using a custom-built system with a high-voltage source connected to the spinneret (stainless steel nozzle) and equipped with a rotating cylindrical-in-shape ground electrode used as a target for harvesting the fibrous deposit, as schematically shown in Figure 3.

Figure 3. Schematic representation of the home-built electrospinning (ES) apparatus.

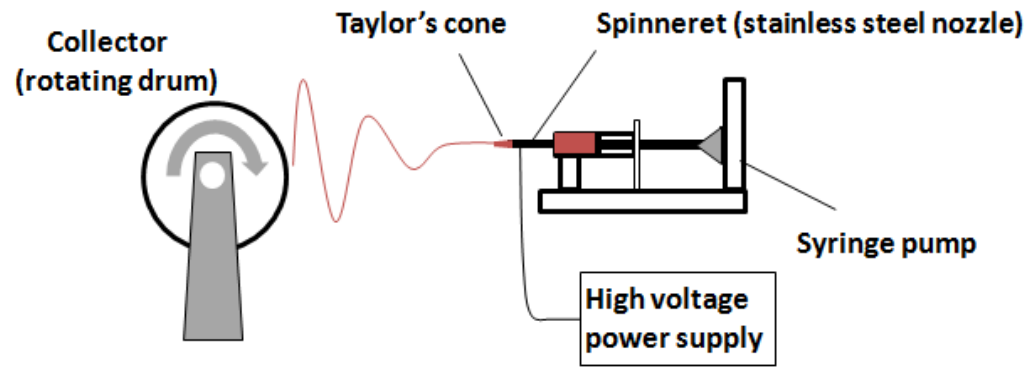

The major instrumental parameters were as follows: the applied "spinneret-collector" voltage up to $15 \mathrm{kV}$ was typically set at $8 \mathrm{kV}$; the "spinneret-collector" distance variable in the range 1 to $15 \mathrm{~cm}$, was typically set at $8 \mathrm{~cm}$; the axial translation of the collector was within the 10-cm range; the solution flow rate was manually adjustable down to $0.1 \mathrm{~mL} / \mathrm{h}$; the rotating collector speed was adjustable in the range of 60 to $1200 \mathrm{rpm}$; the spinneret diameter was of $0.3 \mathrm{~mm}$.

\section{Results and Discussion}

The E-spun fibers formed a dense mat, which could easily be detached from the collector (rotating drum) of our ES apparatus and laid out on a flat surface. The overall morphology of a typical non-woven fabrics obtained via ES processes is shown in Figure 4a.

Figure 4. The morphology of a mat consisting of E-spun polystyrene (PS)-based fibers shown under visible light illumination (a) and near-infrared (NIR) light excitation (b). The rectangular contour marked by the broken green line corresponds to the rectangular shape of the incident NIR laser beam (right panel).

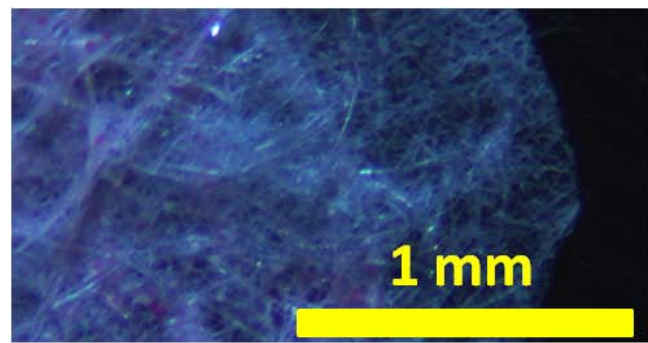

(a)

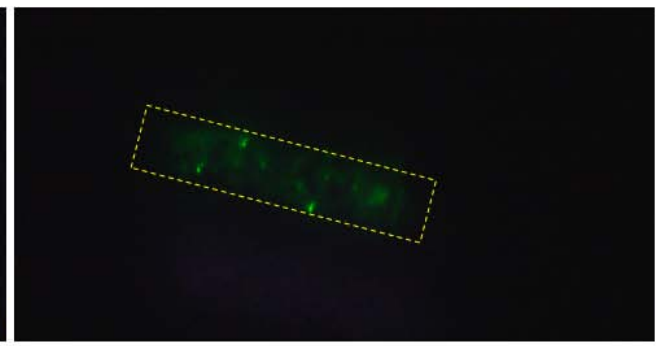

(b) 
As can be seen in Figure 4b, under NIR light excitation $\left(\lambda_{\mathrm{ex}}=975 \mathrm{~nm}\right)$, the non-woven fabric emits a bright yellowish green luminescence, which can be attributed to the sum of two primary emissions bandwidths of the UCNPs, at $525 \mathrm{~nm}$ and $545 \mathrm{~nm}$ (green) and $665 \mathrm{~nm}$ (red), corresponding respectively to the radiative transitions from the ${ }^{2} \mathrm{H}_{11 / 2},{ }^{4} \mathrm{~S}_{3 / 2}$ and ${ }^{4} \mathrm{~F}_{9 / 2}$ excited states to the ${ }^{4} \mathrm{I}_{15 / 2}$ ground state of the $\mathrm{Er}^{3+}$ atom in $\beta-\mathrm{NaYF}_{4}$ : Er, $\mathrm{Yb}$ [37].

The luminescence of the E-spun fibers was checked by positioning a small bundle of fibers in a light integrating sphere (general purpose 6” in diameter integrating sphere, Edmund Optics Ltd., York, UK), which was coupled via an optical fiber to a spectrofluorometer, model USB2000+XR1 (Ocean Optics, Dunedin, FL, USA). The NIR light excitation $\left(\lambda_{\mathrm{ex}}=975 \mathrm{~nm}\right)$ was performed using a 2-W variable power laser diode, model MDL-III-975 (GMP SA, Renens, Switzerland). The evolution of the luminescence spectra as a function of the NIR light excitation power for the PS-based fibers loaded with UCNPs and RB are shown in Figure 5.

Figure 5. The evolution of the luminescence spectra acquired for the PS-based fibers containing upconversion nanophosphors (UCNPs) and rose bengal (RB) as a function of the NIR light excitation power $\left(\lambda_{\mathrm{ex}}=975 \mathrm{~nm}\right)$. Inset: the corresponding log-log plot of the green luminescence versus the excitation power.

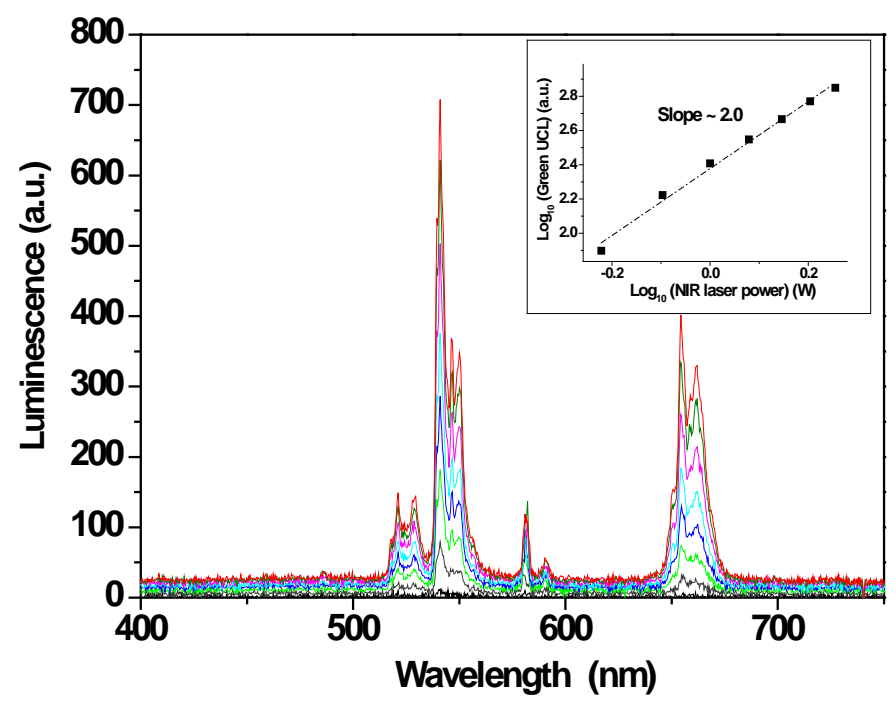

The luminescence spectra shown in Figure 5 originate from the upconversion process and are characteristic for $\beta$-NaYF 4 -based UCNPs [37]. In particular, the slope of $\sim 2.0$ derived for the log-log plot of the green luminescence (integrated UCL bandwidths of the UCNPs at $525 \mathrm{~nm}$ and $545 \mathrm{~nm}$ ) versus the excitation power is similar to the slope values previously reported for the luminescence of $\beta-\mathrm{NaYF}_{4} \mathrm{UCNPs}$ under NIR light excitation and points to the fact that the radiative transitions in UCNPs embedded in the PS matrix of the E-spun fibers follow the two-photon upconversion process (inset to Figure 5) [37,46].

A more detailed insight into the morphology of E-spun PSFs was gained by performing SEM and AFM imaging. The typical SEM images of PSFs are shown in Figure 6. As can be seen in this figure, the individual E-spun fibers reveal high structural quality and high porosity. Moreover, the accomplished 
SEM imaging and associated elemental analysis based on energy dispersive X-ray spectroscopy (EDX) of the E-spun fibers pointed to the presence of both UCNPs and SPIONs entrapped in the PS matrix (Figure 7).

Figure 6. The morphology of the E-spun polystyrene-based fibers (PSFs) containing co-encapsulated UCNPs and SPIONs: (a) SEM image of the fiber network; (b) SEM image of an individual fiber.

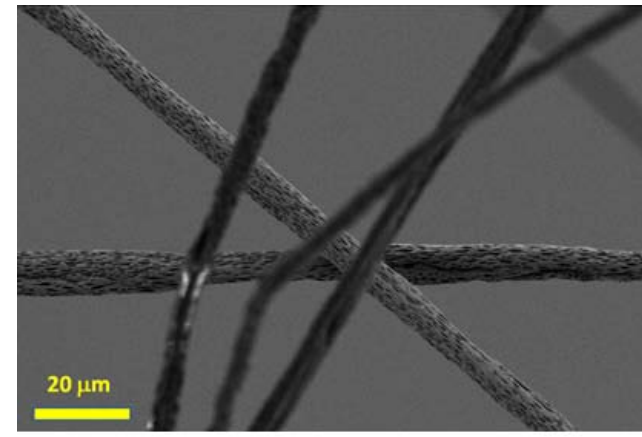

(a)

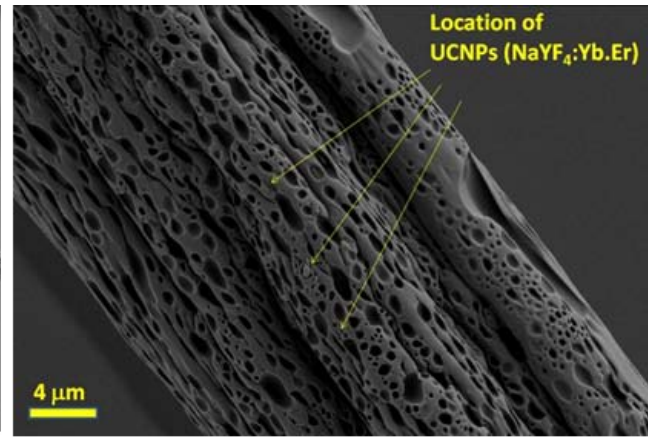

(b)

Figure 7. The SEM image of the surface of the E-spun PS fiber and the corresponding X-ray spectroscopy (EDX) spectra pointing to the presence of SPIONs (upper spectrum) and UCNPs (lower spectrum).

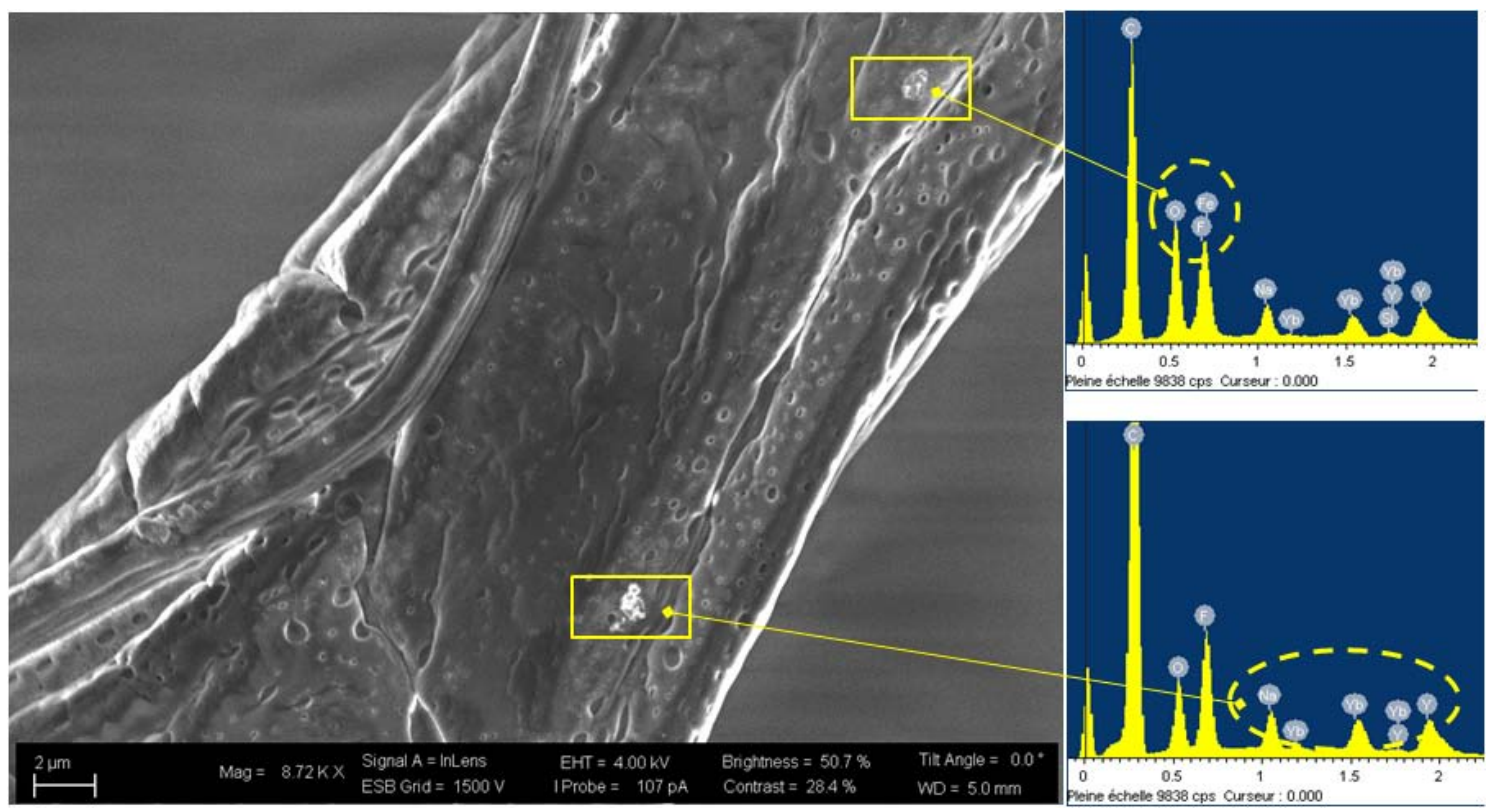

As can be seen in Figure 7, the typical EDX spectra acquired for E-spun PSFs revealed well-defined diffraction peaks characteristic for iron atoms in SPIONs and sodium, yttrium and ytterbium atoms in UCNPs.

The accomplished AFM imaging of individual fibers confirmed their high porosity, with an average pore depth of $c a$. 50-70 nm (Figure 8).

The magnetic properties of PS-based E-spun fibers loaded with UCNPs and SPIONs were checked in ESR experiments. Room temperature ESR measurements were performed for the original ferro-fluid containing the suspension of $\gamma-\mathrm{Fe}_{2} \mathrm{O}_{3}$ superparamagnetic particles (aqueous suspension, $10 \mathrm{mg}$ of Fe per 1 
$\mathrm{mL}$, at $\mathrm{pH}$ 2) and for the E-spun fibers. The ESR spectra were acquired using a Bruker ESP300E spectrometer operating at the microwave frequency $9.7 \mathrm{GHz}$ (X-band). As can be seen in Figure 9, the ESR spectrum acquired for SPIONs embedded in the PS-based E-spun fibers broadens considerably as compared to the spectrum of SPIONs in the original ferro-fluid. The corresponding spectral line widths, $\Delta \mathrm{H}_{\mathrm{pp}}$, are of $\sim 600 \mathrm{G}$ and $\sim 1110 \mathrm{G}$, for SPIONs in ferro-fluid and PS fibers, respectively. This linebroadening can be ascribed to a stronger immobilization of $\gamma-\mathrm{Fe}_{2} \mathrm{O}_{3}$ superparamagnetic particles in large fragments of PS-based fibers. This observation is in good agreement with the previous reports on SPIONs immobilized in bulk polymer matrices [47].

Figure 8. Atomic force microscopy (AFM) characterization of the PS-based E-spun fibers: AFM image of the surface of the E-spun fiber (a); and AFM-derived profiles of pores on the surface of the E-spun fiber (b).

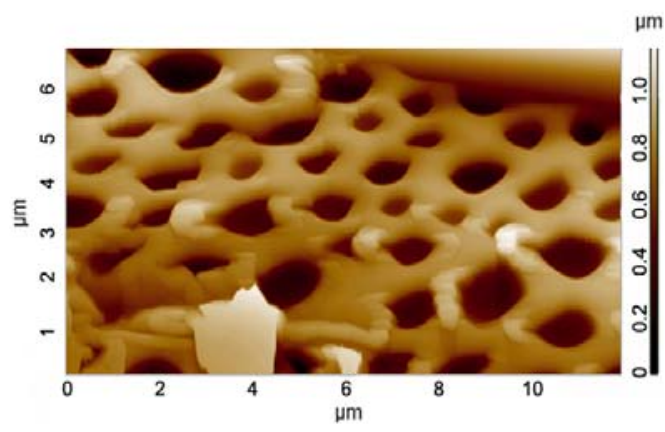

(a)

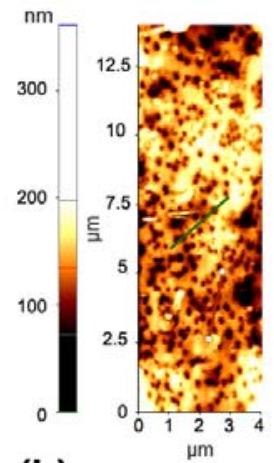

(b)

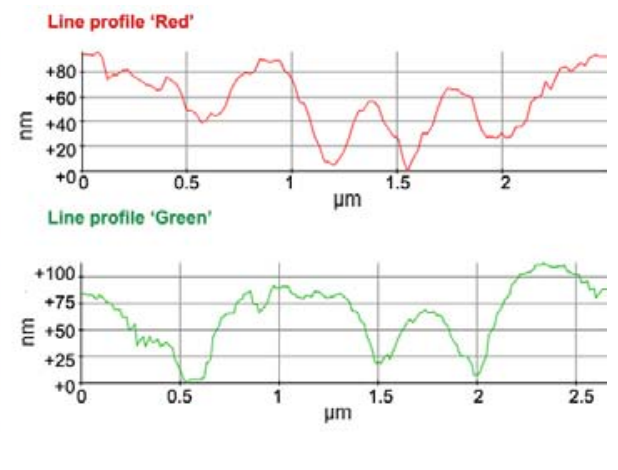

Figure 9. The comparison of the electron spin resonance (ESR) spectra acquired for SPIONS present in the original ferro-fluid (blue trace) and in the E-spun PS fibers loaded with UCNPs and SPIONs (red trace).

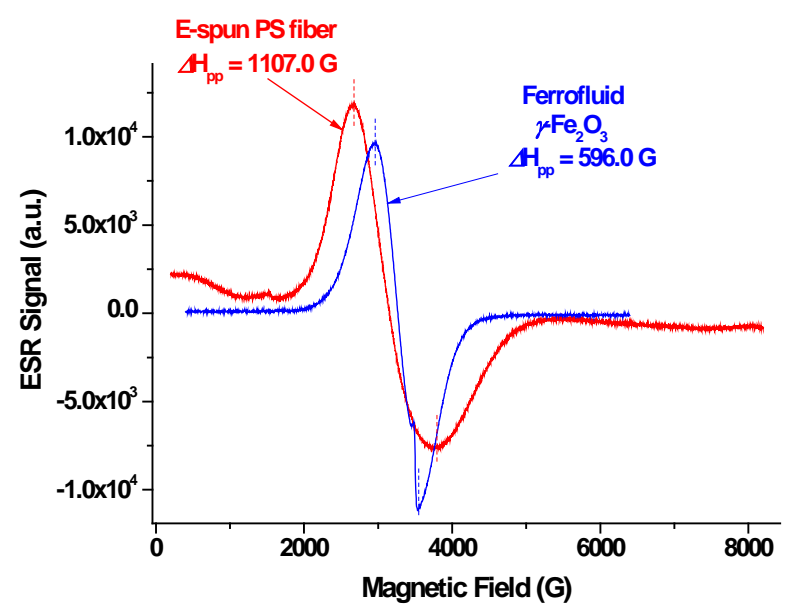

The photodynamic action of the upconversion nanophosphors towards the generation of singlet oxygen under the excitation with NIR light was first verified for the aqueous suspensions of ground UCNPs. To generate ${ }^{1} \Delta_{\mathrm{g}}$ in aqueous suspensions of UCNPs, we employed a water-soluble ${ }^{1} \Delta_{\mathrm{g}}$-sensitizer, rose bengal (RB), from Sigma-Aldrich. The rationale of using $\mathrm{RB}$ as the ${ }^{1} \Delta_{\mathrm{g}}$ photosensitizer in conjunction with 
UCNPs is explained in Figure 10. The example absorption spectrum of $13 \mu \mathrm{M}$ RB in $\mathrm{H}_{2} \mathrm{O}$ (pink trace in Figure 10) was measured with a UV-Vis spectrophotometer, Cary 50 Bio, Varian, Australia. The example UCL spectrum of UCNPs is also shown in this figure (green trace). As can be seen, the spectral absorption of RB perfectly matches the green emission region of UCNPs. Thus, this result confirms the rationale of using $\mathrm{RB}$ as the ${ }^{1} \Delta_{\mathrm{g}}$ photosensitizer in conjunction with $\beta$-NaYF $\mathrm{F}_{4}$ :Yb,Er based UCNPs.

Figure 10. The overlapped spectra of the upconversion luminescence of UCNPs (green trace) and of the absorbance spectrum of $13-\mu \mathrm{M}$ water solution of rose bengal (pink trace). The UCNPs were suspended in water at a concentration of $0.5 \mathrm{mg} / \mathrm{mL}$.

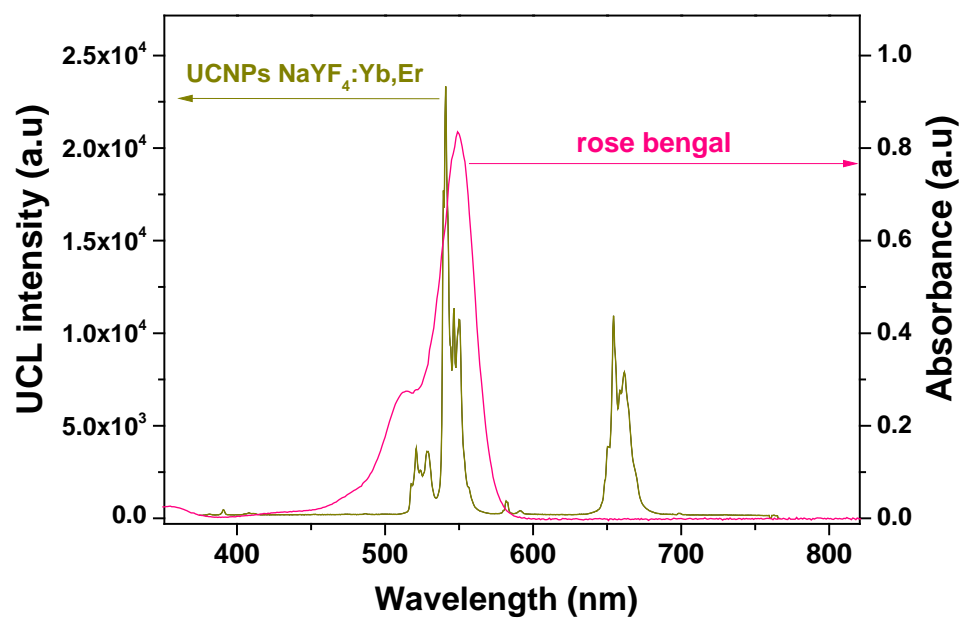

The ESR technique was used to follow the formation of the photosensitized singlet oxygen in aqueous suspensions of UCNPs. The experiments were performed in deuterated water $\left(\mathrm{D}_{2} \mathrm{O}\right)$, which markedly enhances the singlet oxygen lifetime, from $3.1 \mu$ s in $\mathrm{H}_{2} \mathrm{O}$ to $68 \mu$ s in $\mathrm{D}_{2} \mathrm{O}$ [48]. The UCNPs were suspended in $\mathrm{D}_{2} \mathrm{O}$ by sonication. After sonication, the $\mathrm{D}_{2} \mathrm{O}$ suspensions of UCNPs were mixed with $\mathrm{D}_{2} \mathrm{O}$ solutions containing RB and a diamagnetic scavenger of singlet oxygen, 2,2,6,6-tetramethyl-4-piperidinol (TMP-OH). Upon reaction with singlet oxygen, TMP-OH converts to a stable nitroxyl radical, 4-hydroxy2,2,6,6-tetramethylpiperidine-1-oxyl (TEMPOL) [49].

The final suspension contained $2 \mathrm{mg} / \mathrm{mL}$ of UCNPs, $50 \mu \mathrm{M}$ concentration of $\mathrm{RB}$ and $50 \mathrm{mM}$ concentration of TMP-OH. The 2-mL volume of this suspension was then transferred into a small Pyrex beaker ( $5 \mathrm{~mL}$ ), which was positioned in the thermostated water bath (set to $25^{\circ} \mathrm{C}$ ) of the customdesigned photoreactor. To generate ${ }^{1} \Delta_{\mathrm{g}}$, the sample was exposed to NIR light from a 2-W NIR laser diode operating at $975 \mathrm{~nm}$ (MDL-III-975, GMP SA, Renens, Switzerland). The output of the NIR laser was positioned above the beaker, at a distance of $5 \mathrm{~cm}$ from the sample, thus providing the power density of $\sim 5.0 \mathrm{~W} / \mathrm{cm}^{2}$. During illumination with NIR light, the suspensions were magnetically stirred to prevent the precipitation of UCNPs. The experimental setup is shown in Figure 11. 
Figure 11. Photographs of the custom-made photoreactor for performing NIR light stimulated generation of singlet oxygen in aqueous suspensions of UCNPs: (a) visible light image; (b) image taken under illumination of the sample with NIR light in the absence of rose bengal; (c) image taken under illumination of the sample with NIR light in the presence of rose bengal.

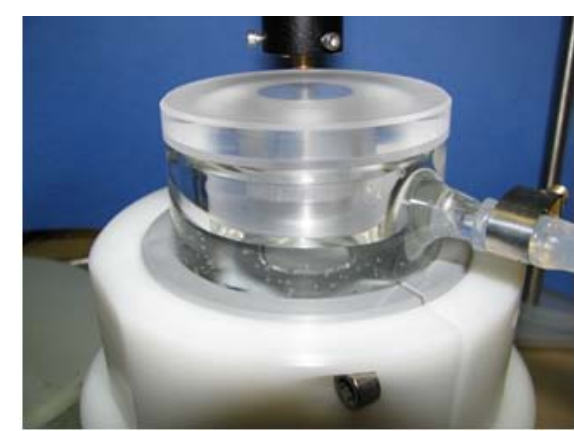

(a)

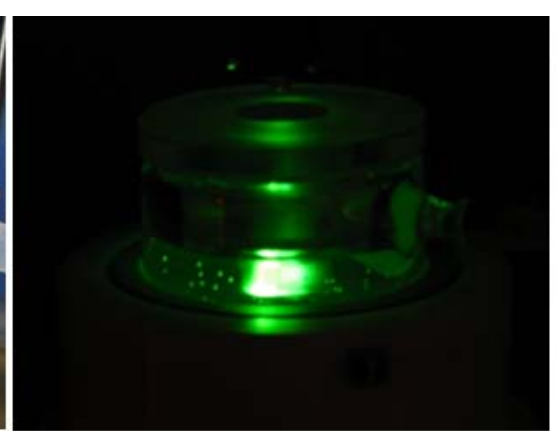

(b)

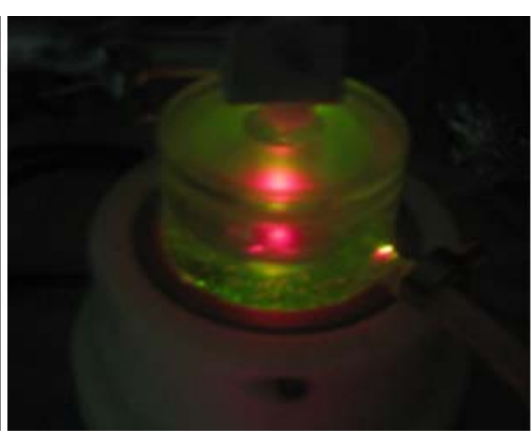

(c)

After subsequent illumination steps, small aliquots of 15 microliters were transferred into $0.7 \mathrm{~mm}$ inner diameter and $0.87 \mathrm{~mm}$ outer diameter glass capillary tubes (VitroCom, Mountain Lakes, NJ, USA), with a sample height of $\sim 40 \mathrm{~mm}$, and sealed on both ends with a tube sealing compound (Cha-seal from Chase Scientific Glass, Rockwood, TN, USA) for performing ESR measurements. To maximize the sample volume in the active zone of the ESR cavity, assemblies of seven tightly packed capillaries were bundled together and inserted into the wide-bore quartz capillary (standard ESR quartz tube with $2.9 \mathrm{~mm}$ ID and 4 mm OD, Model 707-SQ-250M, from Wilmad-LabGlass Inc., Vineland, NJ, USA). Such a setup resulted in a 65 microliter sample volume in the active zone of the rectangular $\mathrm{TE}_{102}$ cavity.

As can be seen in Figure 11c, under NIR light excitation, the bright yellowish green luminescence, which is typical for $\mathrm{NaYF}_{4}$ :Yb,Er nanophosphors, changes its color, due to a partial absorption of the green UCL by the photosensitizer (RB). This confirms the energy transfer from the NIR light excited UCNPs to RB.

The ESR results of singlet oxygen detection under NIR light excitation of aqueous suspensions containing UCNPs and the ${ }^{1} \Delta_{\mathrm{g}}$-sensitizer, RB, are shown in Figure 12.

As can be seen in Figure 12, under exposure to NIR light for 90 min, marked concentrations (tens of micromoles) of ${ }^{1} \Delta_{\mathrm{g}}$ were generated in the aqueous suspension containing UCNPs and the ${ }^{1} \Delta_{\mathrm{g}}$-sensitizer, RB.

Finally, we implemented a similar approach to verify by ESR the photodynamic efficiency of the PSbased E-spun fibers towards the generation of singlet oxygen under NIR light illumination in aqueous media. Prior to performing the ESR detection of NIR-light excited singlet oxygen, a small bundle of Espun PS fibers was inserted into a thin glass capillary (0.7 mm ID and $0.87 \mathrm{~mm} \mathrm{OD).} \mathrm{Subsequently,} \mathrm{the}$ capillary was filled with $50 \mathrm{mM}$ solution of TMP-OH in $\mathrm{D}_{2} \mathrm{O}$, sealed on both ends with Cha-seal and inserted into $4.0 \mathrm{~mm}$ OD quartz tube. Then, NIR illumination was performed using a 2-W laser diode operating at $975 \mathrm{~nm}$. After each illumination step, the capillary was positioned in the spectrometer's cavity, and standard field-swept ESR spectra were acquired. The experimental setup is shown in Figure 13. 
Figure 12. The photodynamic process of RB-mediated singlet oxygen generation under NIR light excitation of the $\mathrm{D}_{2} \mathrm{O}$ suspension of UCNPs (black triangles) and the control trace for the $\mathrm{D}_{2} \mathrm{O}$ solution of RB (black hexagons). Insets: (a) typical time evolution of ESR signals of 4hydroxy-2,2,6,6-tetramethylpiperidine-1-oxyl (TEMPOL) during NIR light illumination of the aqueous suspension of UCNPs in the presence of RB; (b) time evolution of ESR signals of TEMPOL during the control measurements of the $\mathrm{D}_{2} \mathrm{O}$ solution of $\mathrm{RB}$ under NIR light excitation (in the absence of UCNPs). In both cases, the concentrations of RB and 2,2,6,6tetramethyl-4-piperidinol (TMP-OH) were of $50 \mu \mathrm{M}$ and $50 \mathrm{mM}$, respectively.

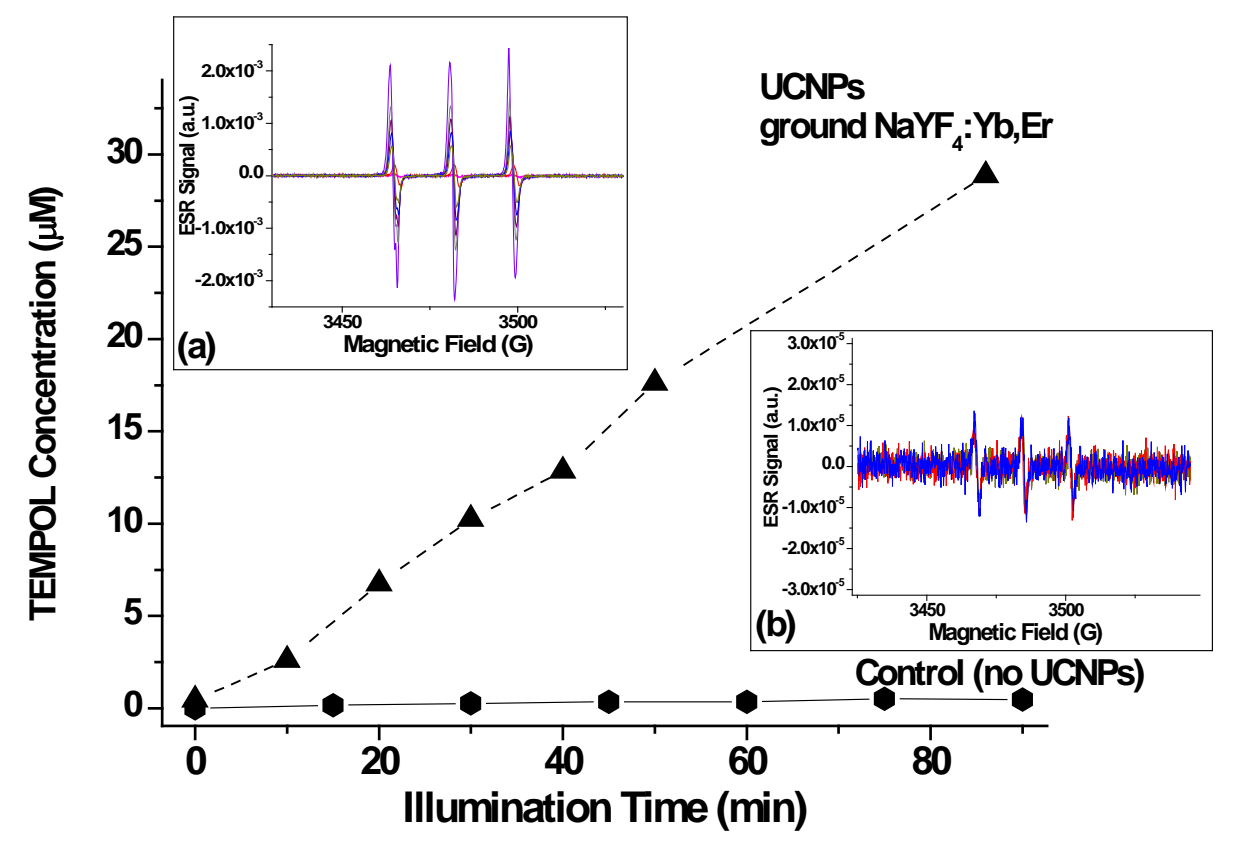

Figure 13. The experimental setup for performing NIR light stimulated the generation of singlet oxygen in the presence of PS-based E-spun fibers immersed in $\mathrm{D}_{2} \mathrm{O}$. (a) The photograph of the termination of the $0.7 \mathrm{~mm}$ inner diameter glass capillary loaded with PS-based fibers and prepared to be filled with the $\mathrm{D}_{2} \mathrm{O}$ solution of TMP-OH; (b) the photograph of the $4.0 \mathrm{~mm}$ outer diameter quartz capillary containing the $0.7 \mathrm{~mm}$ inner diameter glass capillary with PS-based fibers immersed in the $\mathrm{D}_{2} \mathrm{O}$ solution of TMP-OH and positioned under the output of 2-W NIR laser diode $\left(\lambda_{\mathrm{ex}}=975 \mathrm{~nm}\right)$; (c) the photograph showing the luminescence of PS-based fibers positioned in the $0.7 \mathrm{~mm}$ inner diameter glass capillary and exposed to NIR light: the yellowish green UCL of UCNPs embedded in polystyrene fibers can be seen.

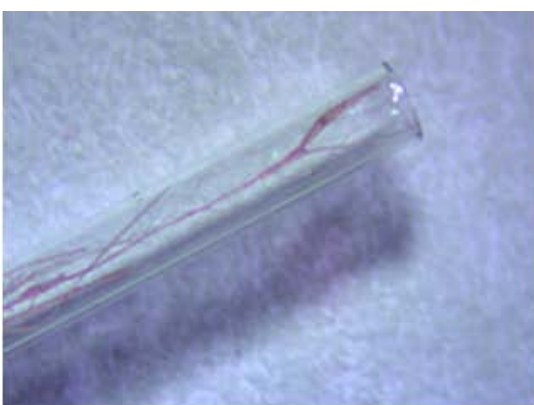

(a)

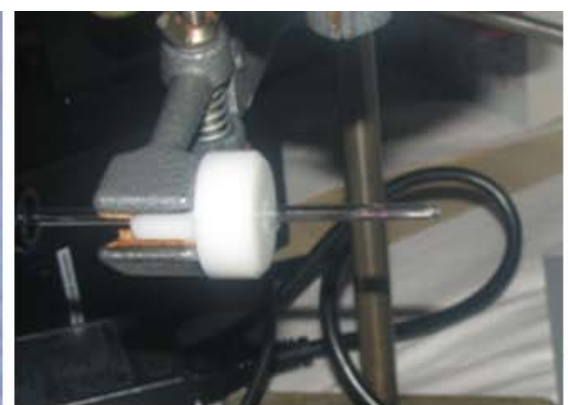

(b)

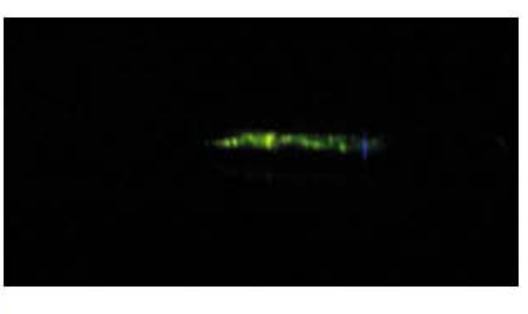

(c) 
Thus, as can be seen in Figure 14, micromolar concentrations of singlet oxygen were photo-sensitized during NIR light illumination for ca. 350 min of PS-based E-spun fibers containing UCNPs, SPIONs and RB. It is worth noting that this result was obtained for a very small total mass of the PS fibers $(\sim 0.2 \mathrm{mg})$ in an aqueous milieu of $\sim 5-6 \mu \mathrm{L}$. We associate this photodynamic activity of the PS-based E-spun fibers with their high porosity and large surface-area-to-volume ratio. The marked slope of the ESR spectra acquired for TEMPOL, which can be seen in inset (a) to Figure 14, is due to a relatively large background signal related to SPIONs entrapped in the E-spun fibers (depicted by the red trace in Figure 9).

Figure 14. The ESR assay of the photodynamic formation of singlet oxygen in $\mathrm{D}_{2} \mathrm{O}$ under the NIR light excitation of PS-based E-spun fibers loaded with UCNPs, SPIONs and RB (black triangles) and the control trace acquired for the undoped PS-based E-spun fibers (black hexagons). In both cases, the PS-based fibers were immersed in $50 \mathrm{mM}$ solution of TMP-OH in $\mathrm{D}_{2} \mathrm{O}$. Inset: (a) the time evolution of the ESR signals of TEMPOL under NIR light illumination of PS-based E-spun fibers loaded with UCNPs, SPIONs and RB; (b) the time evolution of the ESR signals of TEMPOL under NIR light illumination of the undoped PS-based E-spun fibers.

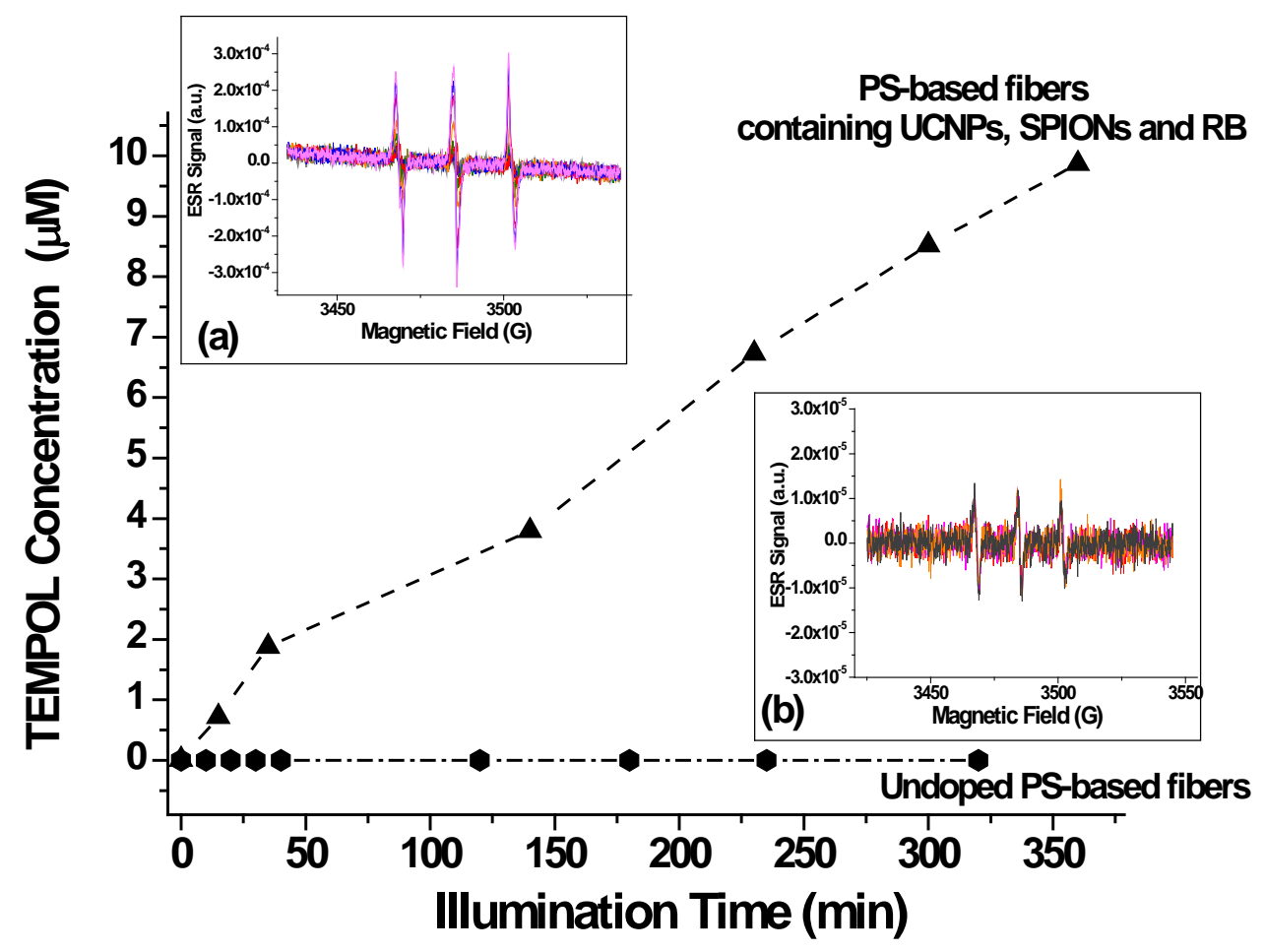

\section{Conclusions}

We successfully prepared PS-based multi-functional magnetic-photoluminescent-photocatalytic microand nano-fibers by a single-step ES process. The fabrication technique was simple, and the materials used were inexpensive. Due to the concomitant loading with UCNPs and RB, the E-spun PS-based fibers revealed photocatalytic activity on exposure to NIR light. In particular, under NIR light excitation, the UCNPs embedded in PS-based fibers emitted the upconversion luminescence (UCL) in the visible range, thus enabling the photosensitization of singlet oxygen by RB. Moreover, due to the doping 
with $\gamma-\mathrm{Fe}_{2} \mathrm{O}_{3}$ SPIONs, these nanostructured PS-based fibers revealed also magnetic properties, typical for the systems containing iron oxide superparamagnetic nanoparticles.

\section{Acknowledgments}

The authors would like to acknowledge the financial support of the Swiss National Science Foundation (SNSF) through the Nano-Tera.ch Focused Project (NTF), "NanoUp”. This work was also partly supported by the Swiss National Science Foundation (SNSF) grant no. PP00P2_123373, the University of Fribourg, and the Adolphe Merkle Foundation (including FriMat). We are grateful to Paul Bowen (Ecole Polytechnique Fédérale de Lausanne) for milling the UCNPs used in this work.

\section{Conflicts of Interest}

The authors declare no conflict of interests.

\section{References}

1. Ladja, R.; Bitar, A.; Eissa, M.M.; Fessi, H.; Mugnier, Y.; Le Dantec, R.; Elaissari, A. Polymer encapsulation of inorganic nanoparticles for biomedical applications. Int. J. Pharm. 2013, 458, 230-241.

2. Peng, W.; Qu, S.; Cong, G.; Wang, Z. Synthesis and structures of morphology-controlled ZnO nanoand microcrystals. Cryst. Growth Des. 2006, 6, 1518-1522.

3. Chatterjee, J.; Bettge, M.; Haik, Y.; Jen Chen, C. Synthesis and characteriza-tion of polymer encapsulated $\mathrm{Cu}-\mathrm{Ni}$ magnetic nanoparticles for hyperthermia applications. J. Magn. Magn. Mater. 2005, 293, 303-309.

4. Hirai, T.; Saito, T.; Komasawa, I. Stabilization of CdS nanoparticles immobilized on thiol-modified polystyrene particles by encapsulation with polythiourethane. J. Phys. Chem. B 2001, 105, 9711-9714.

5. Zeng, Z.; Yu, J.; Guo, Z.-X. Preparation of functionalized core-shell alu-mina/polystyrene composite nanoparticles, 1. Macromol. Chem. Phys. 2005, 206, 1558-1567.

6. Sondi, I.; Fedynyshyn, T.H.; Sinta, R.; Matijevic, E. Encapsulation of nanosizedsilica by in situ polymerization of tert-butyl acrylate monomer. Langmuir 2000, 16, 9031-9034.

7. Venugopal, J.; Ramakrishna, S. Applications of polymer nanofibers in biomedicine and biotechnology. Appl. Biochem. Biotechnol. 2005, 125, 147-157.

8. Khetani, S.R.; Bhatia, S.N. Engineering tissues for in vitro applications. Curr. Opin. Biotechnol. 2005, 17, 524-553.

9. Ravichandran, R.; Gandhi, S.; Sundaramurthi, D.; Sethuraman, S.; Krishnan, U.M. Hierarchical mesoporous silica nanofibers as multifunctional scaffolds for bone tissue regeneration. J. Biomater. Sci. Polym. Ed. 2013, 24, 1988-2005.

10. Focarete, M.L.; Gualandi, C. Potentialities of electrospun polymeric nanofibres in the biomedical field. J. Tissue Sci. Eng. 2013, 4, 1-4.

11. Doshi, J.; Reneker, D.H. Electrospinning process and applications of electrospun fibers. J. Electrost. 1995, 35, 151-160. 
12. Huang, Z.-M.; Zhang, Y.-Z.; Kotaki, M.; Ramakrishna, S. A review on polymer nanofibers by electrospinning and their applications in nanocomposites. Compos. Sci. Technol. 2003, 63, 2223-2253.

13. Oraby, M.A.; Waley, A.I.; El-Dewany, A.I.; Saad E.A.; Abd El-Hady, B.M. Electrospinning of gelatin functionalized with silver nanoparticles for nanofiber fabrication. Model. Numer. Simul. Mater. Sci. 2013, 3, 95-105.

14. El-Assar, M.R.; Fouda, M.M.G.; Kenawy, E.-R. Electrospinning of functionalized copolymer nanofibers from poly(acrylonitrile-co-methyl methacrylate). Adv. Polym. Technol. 2013, 32, 1-11.

15. Sill, T.J.; von Recum, H.A. Electrospinning: Applications in drug delivery and tissue engineering. Biomaterials 2008, 29, 1989-2006.

16. Teo, W.-E.; Inai, R.; Ramakrishna, S. Technological advances in electrospinning of nanofibers. Sci. Technol. Adv. Mater. 2011, 12, 013002:1-013002:19.

17. Kim T.-G.; Ragupathy, D.; Gopalan, A.I.; Lee, K.-P. Electrospun carbon nanotubes-gold nanoparticles embedded nanowebs: Prosperous multi-functional nanomaterials. Nanotechnology 2010, 21, 134021:1-134021:11.

18. Mosinger, J.; Lang, K.; Kubat, P.; Sykora, J.; Hof, M.; Plistil, L.; Mosinger, B. Photofunctional polyurethane nanofabrics doped by zinc tetraphenylporphyrin and zinc phthalocyanine photosensitizers. J. Fluoresc. 2009, 19, 705-713.

19. Mosinger, J.; Lang, K.; Hostomsky, J.; Franc, J.; Sykora, J.; Hof, M.; Kubat, P. Singlet oxygen imaging in polymeric nanofibers by delayed fluorescence. J. Phys. Chem. B 2010, 114, 15773-15779.

20. Mosinger, J.; Lang, K.; Plistil, L.; Josenska S.; Hostomsky, J.; Zelinger, Z.; Kubat, P. Fluorescent polyurethane nanofabrics: A source of singlet oxygen and oxygen sensing. Langmuir 2010, 26, 10050-10056.

21. Li, C.; Lin, J. Rare earth fluoride nano-/microcrystals: Synthesis, surface modification and application. J. Mater. Chem. 2010, 20, 6831-6847.

22. Wang, F.; Liu, X. Recent advances in the chemistry of lanthanide-doped upconversion nanocrystals. Chem. Soc. Rev. 2009, 38, 976-989.

23. Chatterjee, D.K.; Rufalhah, A.J.; Zhang, Y. Upconversion fluorescence imaging of cells and small animals using lanthanide doped nanocrystals. Biomaterials 2008, 29, 937-943.

24. Wang, S.; Feng, J.; Song, S.; Zhang, H. Rare earth fluorides upconversion nanophosphors: From synthesis to applications in bioimaging. CrystEngComm 2013, 15, 7142-7151.

25. Haase, M.; Schafer, H. Upconverting nanoparticles. Angew. Chem. Int. Ed. 2011, 50, 5808-5829.

26. Boyer, J.C.; van Veggel, F.C. Absolute quantum yield measurements of colloidal $\mathrm{NaYF}_{4}: \mathrm{Er}^{3+}, \mathrm{Yb}^{3+}$ upconverting nanoparticles. Nanoscale 2010, 2, 1417-1419.

27. Chen, G.; Qiu, H.; Fan, R.; Hao, S.; Tan, S.; Yang, C.; Han, G. Lanthanide-doped ultrasmall yttrium fluoride nanoparticles with enhanced multicolor upconversion photoluminescence. J. Mater. Chem. 2012, 22, 20190-20196. |

28. Chen, G.; Shen, J.; Ohulchanskyy, T.Y.; Patel, N.J.; Kutikov, A.; Li, Z.; Song, J.; Pandey, R.K.; Ågren, H.; Prasad, P.N.; et al. $\left(\alpha-\mathrm{NaYbF}_{4}: \mathrm{Tm}^{3+}\right) / \mathrm{CaF}_{2}$ core/shell nanoparticles with efficient near-infrared to near-infrared upconversion for high-contrast deep tissue bioimaging. ACS Nano 2012, 6, 8280-8287. 
29. Ostrowski, A.D.; Chan, E.M.; Gargas, D.J.; Katz, E.M.; Han, G.; Schuck, J.; Milliron, D.J.; Cohen, B.E. Controlled synthesis and single-particle imaging of bright, sub-10 nm lanthanide-doped upconverting nanocrystals. ACS Nano 2012, 6, 2686-2692.

30. Shen, J.; Chen, G.; Ohulchanskyy, T.Y.; Kesseli, S.J.; Buchholz, S.; Li, Z.; Prasad, P.N.; Han, G. Tunable near infrared to ultraviolet upconversion luminescence enhancement in $\left(\alpha-\mathrm{NaYF}_{4}: \mathrm{Yb}, \mathrm{Tm}\right) / \mathrm{CaF}_{2}$ core/shell nanoparticles for in situ real-time recorded biocompatible photoactivation. Small 2013, 9, 3213-3217

31. Colombo, M.; Carregal-Romero, S.; Casula, M.F.; Gutierrez, L.; Morales, M.P.; Boehm, I.B.; Heverhagen, J.T.; Prosperi, D.; Parak, W.J. Biological applications of magnetic nanoparticles. Chem. Soc. Rev. 2012, 41, 4306-4334.

32. Laurent, S.; Forge, D.; Port, M.; Roch, A.; Robic, C.; Elst, L.V.; Muller, R.N. Magnetic iron oxide nanoparticles: Synthesis, stabilization, vectorization, physicochemical characterizations, and biological applications. Chem. Rev. 2008, 108, 2064-2110.

33. Zhang, D.; Karki, A.B.; Rutman, D.; Young, D.P.; Wang A.; Cocke, D.; Ho, T.H.; Guo, Z. Electrospun polyacrylonitrile nanocomposite fibers reinforced with $\mathrm{Fe}_{3} \mathrm{O}_{4}$ nanoparticles: Fabrication and property analysis. Polymer 2009, 50, 4189-4198.

34. Zhu, J.; Wei, S.; Rutman, D.; Haldolaarachchige, N.; Young, D.P.; Guo, Z. Magnetic polyacrylonitrile-Fe@FeO nanocomposite fibers-electrospinning, stabilization and carbonization. Polymer 2011, 52, 2947-2955.

35. De Santis, R.; Gloria, A.; Russo, T.; D’Amora, U.; Zeppetelli, S.; Dionigi, C.; Sytcheva, A.; Herrmannsdörfer, T.; Dediu, V.; Ambrosio, L. A basic approach toward the development of nanocomposite magnetic scaffolds for advanced bone tissue engineering. J. Appl. Polym. Sci. 2011, 122, 3599-3605.

36. Meng, J.; Xiao, B.; Zhang, Y.; Liu, J.; Xue, H.; Lei, J.; Kong, H.; Huang, Y.; Jin, Z.; Gu, N.; et al. Super-paramagnetic responsive nanofibrous scaffolds under static magnetic field enhance osteogenesis for bone repair in vivo. Nat. Sci. Rep. 2013, 3, 2655. doi:10.1038/srep02655.

37. Suyver, J.F.; Grimm, J.; van Veen, M.K.; Biner, D.; Kramer, K.W.; Gudel, H.U. Upconversion spectroscopy and properties of $\mathrm{NaYF}_{4}$ doped with $\mathrm{Er}^{3+}, \mathrm{Tm}^{3+}$ and/or $\mathrm{Yb}^{3+}$. J. Lumin. 2006, 117, $1-12$.

38. Ye, X.C.; Collins, J.E.; Kang, Y.J.; Chen, J.; Chen, D.T.N.; Yodh, A.G.; Murray, C.B. Morphologically controlled synthesis of colloidal upconversion nanophosphors and their shape-directed self-assembly. Proc. Natl. Acad. Sci. USA 2010, 107, 22430-22435.

39. Gryglik, D.; Lach, M.; Miller, J.S. The aqueous photosensitized degradation of butylparaben. Photochem. Photobiol. Sci. 2009, 8, 549-555.

40. DeRosa, M.C.; Crutchley, R.J. Photosensitized singlet oxygen and its applications. Coord. Chem. Rev. 2002, 233-234, 351-371.

41. Liu, X.; Zhang, Y.; Tu, L.; Wang, Y.; Zeng, Q.; Li, C.; Shi, Z.; Kong, X.; Zhang, H. Breakthrough in concentration quenching threshold of upconversion luminescence via spatial separation of emitter doping area for Bio-application. Chem. Commun. 2011, 47, 11957-11959.

42. Soukka, T.; Kuningas, K.; Rantanen, T.; Haaslahti, V.; Lovgren, T. Photochemical characterization of up-converting inorganic lanthanide phosphors as potential labels. J. Fluoresc. 2005, 15, 513-528. 
43. Bee, A.; Massart, R.; Neveau, S. Synthesis of very fine maghemite particles. J. Magn. Magn. Mater. 1995, 149, 6-9.

44. Van Ewijk, G.A.; Vroege, G.J.; Philipse, A.P. Convenient preparation methods for magnetic colloids. J. Magn. Magn. Mater. 1999, 201, 31-33.

45. Chastellain, M.; Petri, A.; Hofmann, H. Particle size investigations of a multistep synthesis of PVA coated superparamagnetic nanoparticles. J. Colloid Interface Sci. 2004, 278, 353-360.

46. Lim, S.F.; Ryu, W.S.; Austin, R.H. Particle size dependence of the dynamic photophysical properties of $\mathrm{NaYF}_{4}: \mathrm{Yb}$, Er nanocrystals. Opt. Express 2010, 18, 2309-2316.

47. Noginova, N.; Chen, F.; Weaver, T.; Giannelis, E.P.; Bourlinos, A.B.; Atsarkin, V.A. Magnetic resonance in nanoparticles: Between ferro- and paramagnetism. J. Phys.: Condens. Matter 2007, 19, 246208:1-246208:15.

48. Schweitzer, C.; Schmidt, R. Physical mechanisms of generation and deactivation of singlet oxygen. Chem. Rev. 2003, 103, 1685-757.

49. Sienkiewicz, A.; Vileno, B.; Pierzchala, K.; Czuba, M.; Marcoux, P.; Graczyk, A.; Fajer, P.G.; Forro, L. Oxidative stress-mediated protein conformation changes: ESR study of spin-labelled staphylococcal nuclease. J. Phys.: Condens. Matter 2007, 19, 285201:1-285201:13.

(C) 2014 by the authors; licensee MDPI, Basel, Switzerland. This article is an open access article distributed under the terms and conditions of the Creative Commons Attribution license (http://creativecommons.org/licenses/by/3.0/). 